\title{
CHRONIC OBSTRUCTIVE PULMONARY DISEASE
}

\section{Health status measurement: sensitivity of the self-reported Chronic Respiratory Questionnaire (CRQ-SR) in pulmonary rehabilitation}

\author{
J E A Williams, S J Singh, L Sewell, M D L Morgan
}

Thorax 2003;58:515-518

See end of article for authors' affiliations

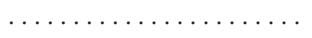

Correspondence to: JE A Williams, Department of Respiratory Medicine, University Hospitals of Leicester NHS Trust Glenfield Hospital, Groby Road, Leicester LE3 9QP, UK; johanna.williams@ uhl-tr.nhs.uk

Revised version received 13 January 2003

Accepted for publication 2 February 2003
Background: A self-reported $C R Q$ (CRQ-SR) has recently been developed and found to be a reproducible and reliable measure of health status. This study explores both the sensitivity of the $C R Q-S R$ and relative sensitivity compared with the conventional interviewer led CRQ (CRQ-IL) in patients undergoing pulmonary rehabilitation.

Methods: Eighty patients with stable chronic obstructive pulmonary disease who had been referred for pulmonary rehabilitation completed the CRQ-SR at initial assessment and at the end of the 7 week programme. A further 35 patients completed both the CRQ-SR and the CRQ-IL, administered 1 week apart, before starting rehabilitation and again at the end of the programme.

Results: There were large statistically and clinically significant changes in mean score per dimension following rehabilitation in all dimensions of the CRQ-SR (dyspnoea mean difference $0.87195 \% \mathrm{Cl}$ 0.61 to 1.14 ); fatigue 0.76 (0.53 to 1.0); emotion 0.60 (0.35 to 0.86$)$; mastery 0.76 (0.52 to 1.0); $\mathrm{p}<0.001)$. Similar results were found in the comparison of the sensitivity of the CRQ-SR and the CRQIL, with large changes in mean score per dimension following rehabilitation for both versions of the questionnaire $(p<0.005)$. No significant differences were seen in the magnitude of change between the two formats of the questionnaire ( $p>0.05)$.

Conclusion: The self-reported $C R Q$ is as sensitive to change as the interviewer led $C R Q$ in patients undergoing pulmonary rehabilitation but has the advantage of being less time consuming to administer.
A ssessment of health status has become an important outcome for treatment in chronic obstructive pulmonary disease (COPD). There is a need for health status measures that are practical and easy to use, both in research studies and also increasingly in the evaluation of clinical services.

The Chronic Respiratory Questionnaire (CRQ) is a well established measure of health status that is both reproducible $^{1-3}$ and sensitive to change. ${ }^{4-8}$ However, as an interviewer led questionnaire the CRQ is time consuming to administer, with an initial interview taking up to half an hour to complete. We have developed a self-reported version of the CRQ that has been found to be reproducible and reliable but is also quick and easy to administer.' The purpose of this study was to examine the sensitivity to change of the self-reported CRQ (CRQ-SR) in a group of subjects undergoing pulmonary rehabilitation. In addition the relative sensitivity of the CRQ-SR was compared with that of the interviewer led CRQ (CRQ-IL).

\section{METHODS}

Sensitivity of the CRQ-SR

The development of the CRQ-SR has been described previously. ${ }^{9}$ The CRQ, and the subsequent CRQ-SR, is made up of four dimensions relating to dyspnoea, emotional function, fatigue, and mastery. There are 20 questions in total and for every question there is a range of responses that score from 1 to 7 . The dimensions of fatigue, emotional function, and mastery are standardised whereas the dyspnoea component is not standardised. Patients are required to identify everyday activities that make them breathless and then select, rank, and score the five most important activities on the seven point scale. Every patient will have a unique list of activities. In each dimension the lower the score, the greater the degree of dysfunction.

Eighty patients with stable COPD who had been referred for pulmonary rehabilitation were recruited for the study. They were given the CRQ-SR to complete at the initial assessment for the programme. Simple verbal instructions were given for filling in the questionnaire and patients completed the questionnaire at home. Patients were informed that if they had difficulty filling in any sections they were to leave them blank and would be given assistance to complete it when they handed it in. Patients also performed the incremental shuttle walk test (ISWT) at this time ${ }^{10}$ and forced expiratory volume in 1 second $\left(\mathrm{FEV}_{1}\right)$ was recorded. Patients then commenced a course of pulmonary rehabilitation, usually starting 1 week after assessment. The CRQ-SR was handed in at the start of the course and any sections missing or not filled in correctly were addressed at this time.

The duration of the pulmonary rehabilitation programme was twice a week for 7 weeks. The exercise component consisted of individually prescribed endurance walking training and a circuit of upper and lower limb strength training exercises. Education sessions included physiotherapy management (relaxation, breathing control and chest clearance), disease education, nutrition, medication and inhaler technique, energy conservation, exacerbation management, and a patient support group.

At the penultimate session of the course patients were given the CRQ-SR to fill in at home and asked to return it at the next session (the dyspnoea provoking activities identified from the initial questionnaire were transcribed onto the follow up questionnaire but subjects were unaware of their previous responses). On discharge, patients performed the ISWT again and were given advice on a maintenance exercise programme. 
Table 1 Sensitivity of the CRQ-SR: mean score per dimension at baseline and after rehabilitation $(n=80)$

\begin{tabular}{lllll}
\hline Dimension & Baseline & $\begin{array}{l}\text { After } \\
\text { rehabilitation }\end{array}$ & $\begin{array}{l}\text { Mean difference } \\
(95 \% \mathrm{Cl})^{*}\end{array}$ & p value† \\
\hline Dyspnoea & $2.38(0.98)$ & $3.25(1.36)$ & $0.87(0.61$ to 1.14$)$ & $<0.001$ \\
Fatigue & $3.13(1.16)$ & $3.89(1.22)$ & $0.76(0.53$ to 1.00$)$ & $<0.001$ \\
Emotion & $4.04(1.32)$ & $4.64(1.25)$ & $0.60(0.35$ to 0.86$)$ & $<0.001$ \\
Mastery & $3.98(1.32)$ & $4.74(1.41)$ & $0.76(0.52$ to 1.00$)$ & $<0.001$ \\
\hline
\end{tabular}

All values are presented as mean (SD).

* Mean difference with $95 \%$ confidence interval $(\mathrm{Cl})$ between pre and post CRQ-SR

tp value of difference between pre and post CRQ-SR from Wilcoxon tests.

\section{Comparison of the sensitivity of the CRQ-SR with the CRQ-IL}

A further 35 patients completed both the CRQ-SR and the interviewer led CRQ before and on completion of the rehabilitation programme. (These subjects are part of a cohort of 52 patients who are described in a previous study where the CRQ-IL was compared with the CRQ-SR before commencing rehabilitation ${ }^{9}$ ). The CRQ-IL was administered at the time of initial assessment for pulmonary rehabilitation. One week later patients were given the CRQ-SR to complete at home. Patients were not informed of the dyspnoea choices they had selected previously on the CRQ-IL when they filled in the CRQ-SR. Patients then commenced the pulmonary rehabilitation programme. On the last session of the course the CRQ-IL was administered. Patients were then given the CRQ-SR to fill in at home and asked to return the questionnaire to us by mail. For both versions of the CRQ the initial dyspnoea provoking activities were transcribed onto the follow up administration, as is normal practice.

The study was approved by the Leicestershire ethics committee.

\section{Statistical analysis}

The method of scoring is identical for both formats of the questionnaire and the results are presented as mean score per dimension which is obtained by dividing the total score in each dimension by the number of questions in that dimension. A change of 0.5 in each dimension has been calculated for the CRQ-IL as being the minimum change necessary to produce a clinically worthwhile benefit (or minimum clinically important difference (MCID) $)^{11}$ ). We made the assumption that the MCID would be the same for the CRQ-SR as for the CRQ-IL as the basic structure and nature of the questions had not been changed.

As the data are ordinal, Wilcoxon tests were used to evaluate the differences between pre and post rehabilitation scores. However, as the data were also essentially normally distributed and in order to evaluate whether the degree of change

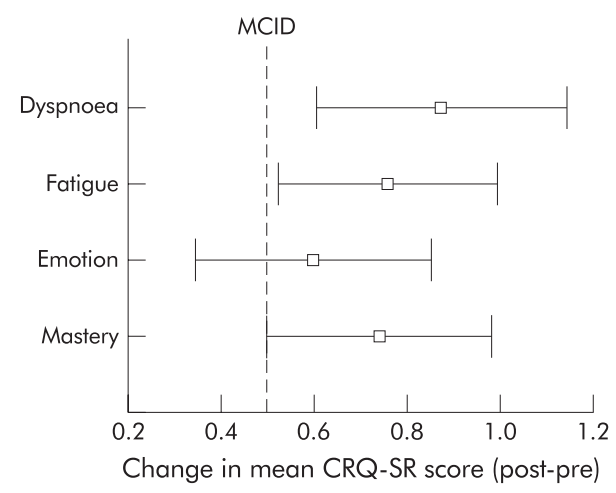

Figure 1 Plot comparing mean change in each dimension (post pre rehabilitation) for the CRQ-SR $(n=80)$. Minimum clinically important difference $(M C I D)=0.5$. exceeded the MCID of 0.5 , mean differences and 95\% confidence intervals (CI) are also given for each dimension.

To explore the statistical differences between the CRQ-SR and the CRQ-IL, Wilcoxon tests were used to compare the absolute degree of change following rehabilitation between the two formats of the questionnaire. To examine the range of sensitivities and the potential differences in sensitivities between the two formats, limits of agreement ${ }^{12}$ are also presented showing the difference in sensitivity between the CRQ-IL and the CRQ-SR against average sensitivity. A paired $t$ test was used to analyse the difference between baseline and post rehabilitation distance walked in the ISWT. For all analyses a $p$ value of $<0.05$ was considered to be statistically significant. All analyses were performed using the Statistical Package for Social Sciences (SPSS) version 9.0.

\section{RESULTS}

\section{Sensitivity of the CRQ-SR}

Eighty patients (59 men) with moderate to severe COPD of mean (SD) age 67.7 (8.1) years, mean (SD) FEV 0.97 (0.42) l, and mean (SD) ISWT distance 157 (94) metres took part in the study. Table 1 presents the mean scores per dimension at baseline and after rehabilitation for each dimension of the CRQ-SR. Highly statistically significant changes were seen in the scores for each dimension following pulmonary rehabilitation $(\mathrm{p}<0.001)$. The threshold of 0.5 (the MCID) was exceeded in all four dimensions, with the dyspnoea dimension showing the largest change $(0.87)$. In all but the emotional function dimension, even the lower $95 \%$ confidence interval was greater than the MCID, indicating that rehabilitation produced an effect significantly greater than that needed for a minimum clinically worthwhile benefit (fig l).

There was also a highly statistically significant increase in shuttle distance walked after rehabilitation: mean (SD) change 73 (65) metres (95\% CI 58 to 88), p<0.001.

\section{Comparison of the sensitivity of the CRQ-SR and the CRQ-IL}

A further 35 patients (22 men) of mean (SD) age 66.2 (7.7) years, mean (SD) FEV 1.07 (0.56) l, and mean (SD) ISWT distance 183 (102) metres completed both the CRQ-SR and CRQ-IL before and on completion of pulmonary rehabilitation. Table 2 presents the mean scores per dimension at baseline and after rehabilitation for both versions of the questionnaire.

There were highly statistically significant changes after rehabilitation in both the CRQ-SR and the CRQ-IL $(\mathrm{p}<0.005)$. All mean differences were also greater than the MCID of 0.5. There were no statistically significant differences in the magnitude of change after rehabilitation between the CRQ-SR and the CRQ-IL in any dimension $(p>0.05)$. The limits of agreement in each dimension for the difference in sensitivity between the CRQ-IL and the CRQ-SR against the average sensitivity are presented graphically in fig 2 . 
Table 2 Sensitivity of the CRQ-SR and the CRQ-IL: mean scores per dimension at baseline and after rehabilitation for both questionnaires $(n=35)$

\begin{tabular}{|c|c|c|c|c|}
\hline Dimension & Baseline & $\begin{array}{l}\text { After } \\
\text { rehabilitation }\end{array}$ & $\begin{array}{l}\text { Mean difference } \\
(95 \% \mathrm{CI})^{*}\end{array}$ & $\mathrm{p}$ value $\dagger$ \\
\hline \multicolumn{5}{|l|}{ CRQ-SR } \\
\hline Dyspnoea & $2.30(0.70)$ & $3.01(1.07)$ & $0.71 \quad(0.38$ to 1.05$)$ & $<0.001$ \\
\hline Fatigue & $3.10(1.12)$ & $3.85(1.30)$ & 0.75 (0.42 to 1.08$)$ & $<0.001$ \\
\hline Emotion & 4.07 (1.15) & $4.74(1.28)$ & 0.67 (0.35 to 0.99$)$ & $<0.001$ \\
\hline Mastery & $4.23(1.32)$ & $4.91(1.34)$ & $0.68(0.27$ to 1.10$)$ & $<0.005$ \\
\hline \multicolumn{5}{|l|}{ CRQ-IL } \\
\hline Dyspnoea & $2.70(0.80)$ & $3.44(0.83)$ & $0.74(0.47$ to 1.00$)$ & $<0.001$ \\
\hline Fatigue & $3.03(1.05)$ & 3.79 (1.19) & 0.76 (0.43 to 1.07$)$ & $<0.001$ \\
\hline Emotion & 4.35 (1.27) & $4.92(1.22)$ & $0.57(0.21$ to 0.91$)$ & $<0.005$ \\
\hline Mastery & $4.10(1.42)$ & 4.98 (1.19) & $0.88(0.50$ to 1.26$)$ & $<0.001$ \\
\hline
\end{tabular}
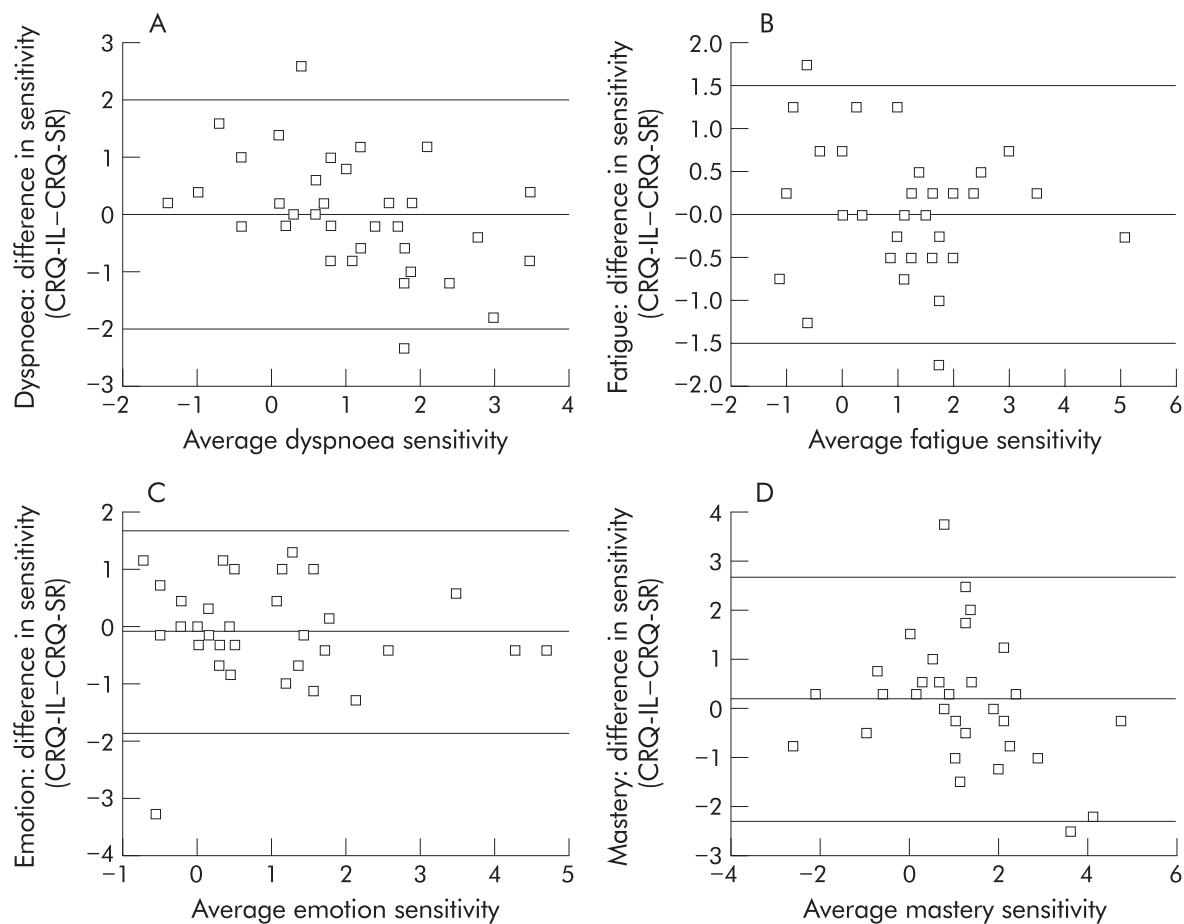

Figure 2 Limits of agreement showing the difference in sensitivity between the CRQ-IL and CRQ-SR against average sensitivity.

\section{DISCUSSION}

This study shows that the CRQ-SR is a sensitive measure of health status in a group of patients with moderate to severe COPD who have undergone a rehabilitation programme. All dimensions of the CRQ-SR showed large statistically significant and clinically important changes after rehabilitation. Our results are in line with a recent meta-analysis carried out by Jones $^{13}$ in which he plotted the results of six studies that have used the CRQ-IL to examine the effect of rehabilitation (drawn from data summarised by Lacasse et $a l^{14}$ ). This meta-analysis showed that mean values in all dimensions of the CRQ-IL improved by a statistically significant amount and were consistently higher than the MCID-that is, pulmonary rehabilitation produced an effect which was significantly greater than that needed for a minimum clinically worthwhile benefit. This was particularly striking for the dyspnoea dimension. Our results indicate a very similar pattern of improvement, again with the dyspnoea dimension showing the most change following rehabilitation. Patients consistently report an improvement in their dyspnoea symptoms fol- lowing rehabilitation, ${ }^{4-8}$ although the mechanism for these improvements is most probably multifactorial, incorporating elements of increased confidence, knowledge of energy conservation principles, increased muscle strength, and cardiovascular fitness. In our study we also found that rehabilitation had a significant effect on walking ability, as measured by the ISWT. However, a minimum clinically important difference for the ISWT has yet to be established.

We also wanted to explore how the two different formats of the CRQ performed when administered to the same group of patients undergoing rehabilitation. In this relatively small cohort of patients we found both formats of the questionnaire showed a similar magnitude of change after rehabilitation. Highly statistically and clinically significant changes were seen in both formats of the questionnaire after rehabilitation, with a very similar magnitude of change for both the CRQ-SR and the CRQ-IL. A weakness of the study was that all patients completed the questionnaires in the same order-that is, CRQ-IL followed by CRQ-SR. However, the questionnaires were administered 7 days apart to a stable population, making the 
possibility of patients remembering their responses less likely and the same conditions applied to the whole cohort.

The CRQ-IL has been used extensively in research trials but it is time consuming to administer. The CRQ-IL can take up to 30 minutes to complete for the initial interview whereas the CRQ-SR takes only 5-10 minutes and can be done in the privacy of a patient's home, thus increasing the chance that patients will answer questions more frankly. ${ }^{15}$ Completion rates for the CRQ-SR were found to be generally high, and any missing information was easily amended when the patient returned the questionnaire. Some subjects (approximately $20 \%$ of our group) found selecting, ranking, and scoring items for the dyspnoea dimension difficult and needed some assistance in completing it. It is recognised that some people may need assistance with this section and this can be done either at the time of initial administration or when the patient returns the questionnaire depending on individual circumstances.

As the previous work by Jones ${ }^{13}$ has highlighted, pulmonary rehabilitation is a very powerful intervention. In this study the CRQ-SR has shown large changes in mean score for all dimensions after rehabilitation. Further work is now needed to establish whether the CRQ-SR is sensitive to change using other interventions and also in other chronic lung conditions.

This study has shown the self-reported CRQ to be as sensitive as the interviewer led CRQ but has the advantage of being less time consuming to administer. The CRQ-SR is thus a sensitive measure of health status in patients with COPD undergoing pulmonary rehabilitation.

\section{Authors' affiliations}

J E A Williams, S J Singh, L Sewell, M D L Morgan, Institute for Lung Health, Department of Respiratory Medicine, University Hospitals of Leicester NHS Trust, Glenfield Hospital, Leicester, UK

Copies of the CRQ-SR can be obtained from the first author.

\section{REFERENCES}

1 Guyatt GH, Berman LB, Townsend M, et al. A measure of quality of life for clinical trials in chronic lung disease. Thorax 1987;42:773-8.

2 Guyatt GH, Townsend M, Keller J, et al. Measuring functional status in chronic lung disease: conclusions from a randomised control trial. Respir Med 1989;83:293-7.

3 Wijkstra PJ, Ten Vergert EM, Van Altena R, et al. Reliability and validity of the chronic respiratory questionnaire (CRQ). Thorax 1994;49:465-7.

4 Guyatt GH, King DR, Feeny DH, et al. Generic and specific measurement of health-related quality of life in a clinical trial of respiratory rehabilitation. J Clin Epidemiol 1999;52:187-92.

5 Singh SJ, Smith DL, Hyland ME, et al. A short outpatient pulmonary rehabilitation programme: immediate and longer term effects on exercise and quality of life. Respir Med 1998;92:1 146-54.

6 Griffiths TL, Burr ML, Campbell IA, et al. Results at 1 year of outpatient multidisciplinary pulmonary rehabilitation: a randomised controlled trial. Lancet 2000;355:362-8.

7 Guell R, Casan P, Belda J, et al. Long term effects of outpatient rehabilitation on COPD: a randomised trial. Chest 2000;117:976-83.

8 Harper R, Brazier JE, Waterhouse JC, et al. Comparison of outcome measures for patients with chronic obstructive pulmonary disease (COPD) in an outpatient setting. Thorax 1997;52:879-87.

9 Williams JEA, Singh SJ, Sewell L, et al. Development of a self-reported Chronic Respiratory Questionnaire (CRQ-SR). Thorax 2001;56:954-9.

10 Singh SJ, Morgan MDL, Scott S, et al. Development of a shuttle walking test of disability in patients with chronic airway obstruction. Thorax 1992:47:1019-24.

11 Redelmeier DA, Guyatt GH, Goldstein RS. Assessing the minimum important difference in symptoms: a comparison of two techniques. J Clin Epidemiol 1996;49:1215-9.

12 Bland JM, Altman DG. Statistical methods for assessing agreement between two methods of clinical measurement. Lancet 1986;i:307-10.

13 Jones PW. Health status measurement in chronic obstructive pulmonary disease. Thorax 2001;56:880-7.

14 Lacasse Y, Wong E, Guyatt GH, et al. Meta-analysis of respiratory rehabilitation in chronic obstructive pulmonary disease. Lancet 1996:348: $1115-9$.

15 Cook DJ, Guyatt GH, Juniper E, et al. Interviewer versus self-administered questionnaires in developing a disease specific, health-related quality of life instrument for asthma. J Clin Epidemiol 1993;46:529-34. 